Document downloaded from:

http://hdl.handle.net/10251/71373

This paper must be cited as:

Balador, A.; Tavares De Araujo Cesariny Calafate, CM.; Cano Escribá, JC.; Manzoni, P. (2013). Congestion Control for Vehicular Environments by Adjusting IEEE 802.11 Contention Window Size. En Algorithms and Architectures for Parallel Processing. Springer. 259-266. doi:10.1007/978-3-319-03889-6_30.

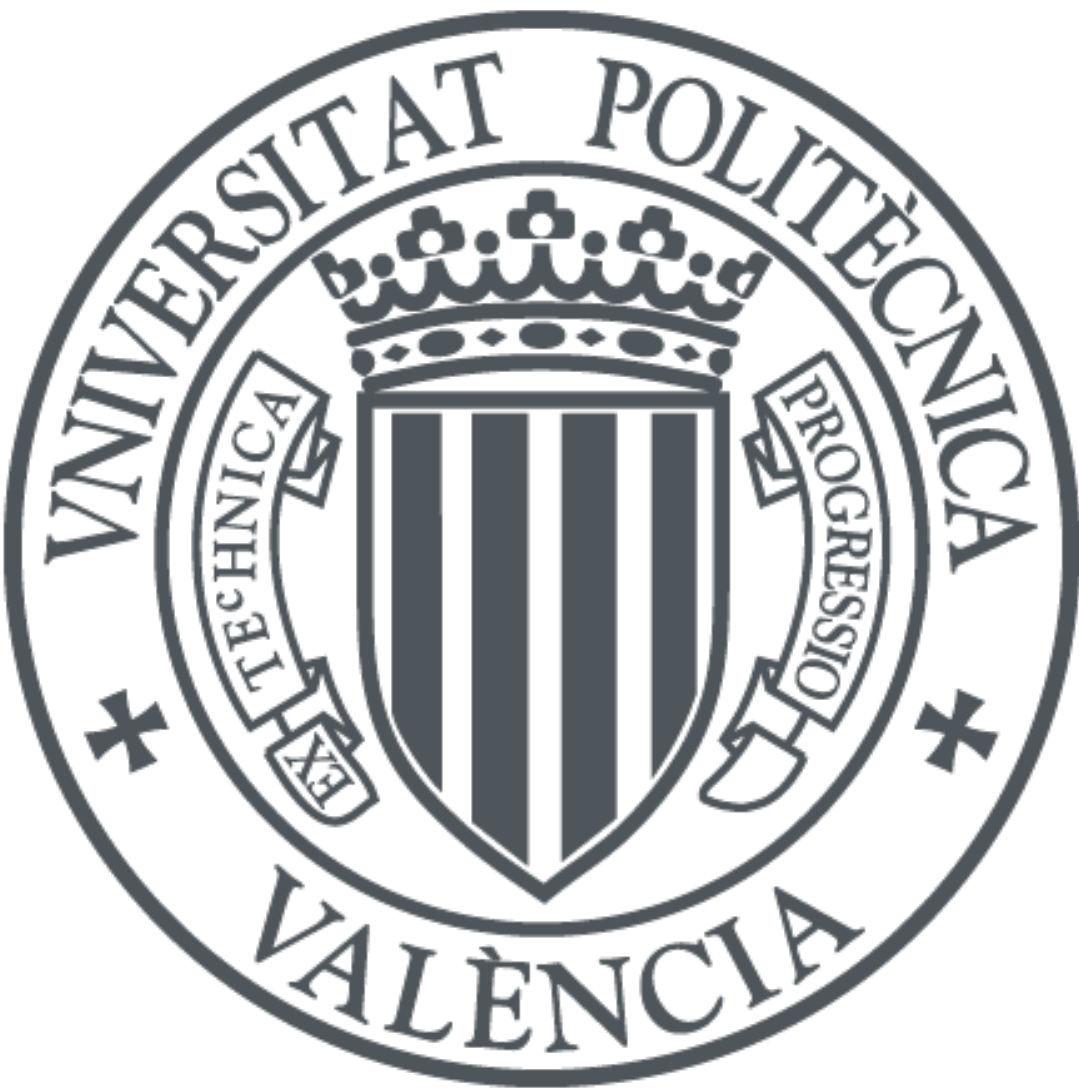

The final publication is available at

http://link.springer.com/chapter/10.1007/978-3-319-03889-6_30

Copyright Springer

Additional Information

The final publication is available at Springer via http://dx.doi.org/10.1007/978-3-319-038896_30 


\title{
Congestion Control for Vehicular Environments by Adjusting IEEE 802.11 Contention Window Size
}

\author{
Ali Balador, Carlos T. Calafate, Juan-Carlos Cano, and Pietro Manzoni \\ Universitat Politecnica de Valencia \\ Camino de Vera, s/n, 46022 Valencia, Spain \\ alba6@upv.es, \{calafate, jucano, pmanzoni\}@disca.upv.es
}

\begin{abstract}
Medium access control protocols should manage the highly dynamic nature of Vehicular Ad Hoc Networks (VANETs) and the variety of application requirements. Therefore, achieving a well-designed MAC protocol in VANETs is a challenging issue. The contention window is a critical element for handling medium access collisions in IEEE 802.11 , and it highly affects the communications performance. This paper proposes a new contention window control scheme, called DBM-ACW, for VANET environments. Analysis and simulation results using OM$\mathrm{NeT}++$ in urban scenarios show that DBM-ACW provides better overall performance compared with previous proposals, even with high network densities.
\end{abstract}

\section{Introduction}

Medium Access Control (MAC) protocols play an important role since critical communications must rely on it. Unfortunately, research results [1] highlight that the topic of MAC support in VANETs has received less attention than in other research fields. Also, most of these relatively few research works are dedicated to V2I communications; therefore, MAC support for V2V communication needs more attention. MAC layer design challenges in VANET environments can be summarized as follows [2]: (a) achieving an effective channel access coordination in the presence of changing vehicle locations and variable channel characteristics; (b) supporting scalability in the presence of various traffic densities; and (c) supporting a diverse set of application requirements.

A lot of research has been done by the research community with the idea of supporting broadcast transmissions in mind (e.g., [3], [4]). In contrast to the most common research trend, this paper targets unicast applications including infotainment, P2P or VoIP.

The IEEE 802.11 has been selected by a wide range of research works for vehicular environments as the MAC layer standard because of its availability, maturity, and cost. However, it causes performance to be poor in VANETs compared to MANET environments. A well-known problem in IEEE 802.11 is scalability, which becomes more challenging in VANETs in the presence of high and 
variable network densities. A lot of works have been proposed and carried out for MANET environments [5], [6], [7] to either solve or reduce this problem.

Among these studies, a dynamic and low-overhead method called HBCWC is proposed in [7]. This method estimates network density based on channel status observations without requiring complex calculations. This way, more than current network status, previous statuses are used in order to identify the channel traffic variations. As a consequence, the $\mathrm{CW}$ size is dynamically tuned based on the estimated network density.

To the best of our knowledge, very few studies address unicast communication in VANETs. A fuzzy logic based enhancement to $802.11 \mathrm{p}$ is proposed in [8] which adapts the CW size based on a non-linear control law, and relies on channel observation. Furthermore, [9] suggests a MAC mechanism which uses a modified version of RTS/CTS in order to estimate network density through message exchange. Therefore, in this paper we propose a new contention window control scheme, called DBM-ACW (Density Based Method for Adjusting the CW size), and prove that DBM-ACW not only outperforms the IEEE 802.11 DCF in different vehicular scenarios, but also previously proposed schemes.

The rest of this paper is organized as follows. In section 2, we describe the new proposed contention window control scheme in detail. Performance evaluation of DBM-ACW, including simulation results in urban scenarios, is presented in section 3. Finally, section 4 concludes this paper.

\section{The Proposed Algorithm}

In order to adequately adjust the $\mathrm{CW}$ size for vehicular environments, we propose DBM-ACW, a new method to select the CW size based on the network traffic density. In this method, the channel condition is estimated based on the packet transmission status, and the result is stored into a Channel State (CS) vector. A significant part of the protocol relies on how the channel conditions are captured by the CS vector, and how this vector is used to update the CW size in order to improve throughput, which it is the key contribution of this paper. These two issues will be further explained in the following sections.

\section{$2.1 \quad$ Initialization}

Similarly to the IEEE 802.11 , the CW value is initially set to $C W_{\min }$, and it is updated during the execution. The CS vector that is used for keeping track of channel conditions is set to one in order to assume a collision free status before starting the simulation. Parameters A and B which are used in Algorithm 2 are set to 1.7 and 0.8 , respectively. Basically, parameters A and B try to optimally adapt the CW size to network density. A more detailed discussion on how these parameters were obtained is presented in the section 2.3. 


\subsection{The Channel State Vector}

In legacy 802.11 DCF, and after each data frame transmission, each node sets its timer and waits for an acknowledgement. In DBM-ACW, upon each timer expiration or upon receiving a packet, Algorithm 1 is called. If the transmitter receives an ACK frame from the receiver, a value of 1 is inserted into the channel state vector (Operation 5). Otherwise, if a collided/faulty frame is received, or if the transmitter waiting timer expires before receiving the acknowledgement, a value of 0 is inserted into the channel vector (Operation 3 ).

The CS vector is updated by shifting after setting the $C S_{0}$ value (Operation 1). The vehicle then calls Adapt (Algorithm 2) through which the $\mathrm{CW}$ is adapted. Based on extensive simulations, we chose a three-element array for DBM-ACW in order to achieve a trade-off between overhead and performance. If we choose a smaller array, it will not be able to reflect the real network conditions, while larger array values do not lead to a significant performance improvement.

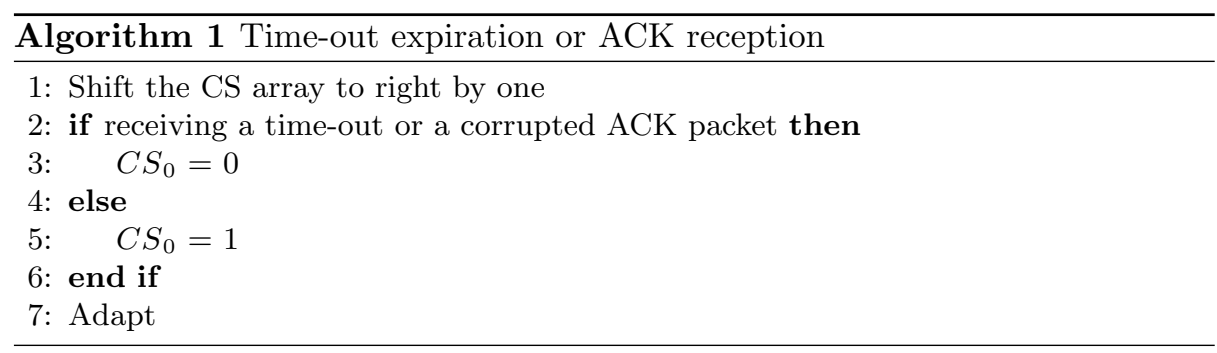

\subsection{Changing the Contention Window Size}

As explained before, upon each timer expiration or packet reception, Adapt (Algorithm 2) is called, in order to update the value of the CW. The CW size is doubled (similarly to the IEEE 802.11 DCF) in order to obtain the highest PDR, except for the case in which the CS array contains two consecutive ones before the new state; in that case the $\mathrm{CW}$ is multiplied by parameter A (Operation 3). Furthermore, the CW size is set to the minimum $\mathrm{CW}, C W_{\min }$, upon each acknowledgement reception, except for the case in which the CS array contains two consecutive zeros before the new state; in that case the $\mathrm{CW}$ is multiplied by parameter B (Operation 9).

The value of parameters A and B in Algorithm 2 was achieved based on extensive simulations in which different combinations of values were used to obtain the best performance. According to the severity of channel congestion, the current $\mathrm{CW}$ size is multiplied by a value in the range from 0.2 to 2 or set to $C W_{\min }$. The upper bound is selected as in the IEEE 802.11 DCF, so that the CW size is multiplied by 2 when the channel is detected as busy or a collision has occurred. When the channel is very congested, the current CW size is multiplied 
by a value in the upper part of this range in order to decrease the probability of selecting the same backoff number. Otherwise, when the channel density is low, the current CW size is multiplied by a value in the lower range or set to $C W_{\min }$ in order to avoid waiting for a long time when the channel occupation is low. In our study, the optimal value for parameters A and B was found to be equal to 1.7 and 0.8 , respectively.

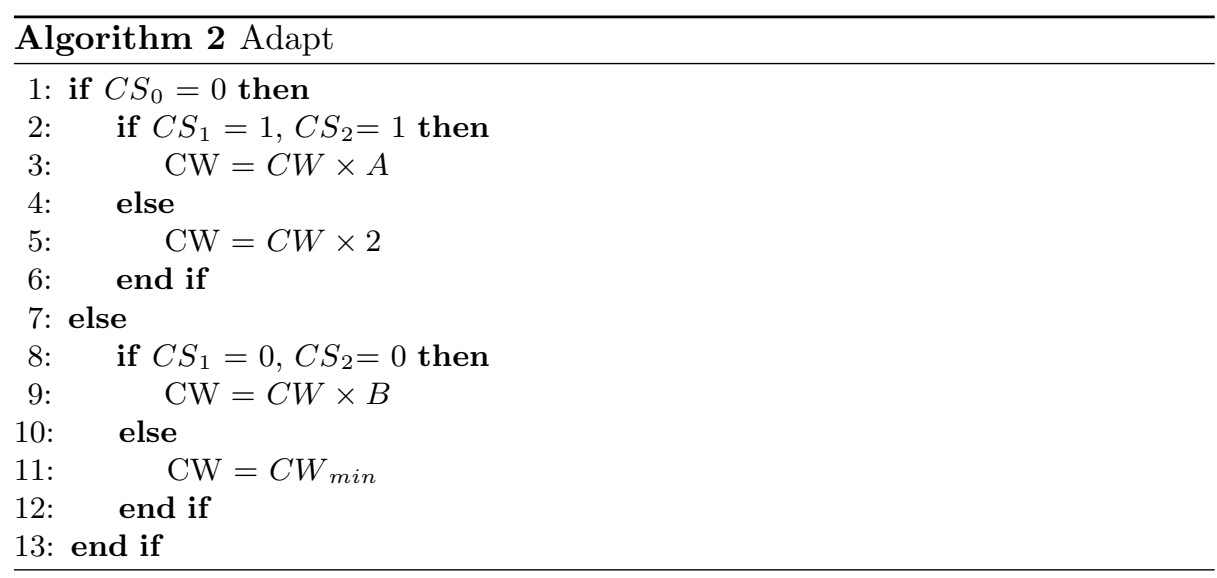

\section{Simulation}

In this section, we study the performance of DBM-ACW in comparison with the IEEE $802.11 \mathrm{DCF}$ and HBCWC in vehicular environments by using OMNeT++ (version 4.2.2) [10]. Therefore, we use this simulator coupled with the INETMANET framework [11] and SUMO [12] in order to provide a realistic vehicular scenario. Also, the VACaMobil [13] tool is used in order to maintain the same number of vehicles throughout the simulation time.

\subsection{Simulation Parameters}

Each vehicle generates constant bitrate traffic. The size of the data payload is 512 bytes, and each node generates data packets at a rate of 4 packets per second. Each vehicle starts a new connection after joining the network, and sends its packets to a randomly selected destination among the current vehicles in the network.

Considering the routing protocol, we assessed different routing protocols (i.e., AODV, OLSR, DYMO, DSR) and, despite of the different overall performance levels obtained by these protocols, we found that they experience the same impact when combined with the different MAC protocols evaluated in this paper. As a consequence, we chose the AODV routing protocol, which is a simple routing 
Table 1: The simulation parameters

\begin{tabular}{ll}
\hline Simulation Parameter & Value \\
\hline \hline Traffic type & CBR \\
CBR packet size & 512 byte \\
CBR data rate & 4 packet/s \\
\hline MAC protocol & $802.11 \mathrm{a}$ \\
Max. and Min. of CW & 7,1023 \\
Max. number of retransmissions & 7 \\
\hline Max. transmission range & $250 \mathrm{~m}$ \\
Propagation model & Nakagami \\
Nakagami-m & 0.7 \\
\hline Simulation time & 300 seconds \\
Number of repetitions & 10 \\
\hline
\end{tabular}

protocol that can be easily implemented when attempting to test it in practical scenarios. The radio propagation range for each node is set to $250 \mathrm{~m}$. We used the Nakagami radio propagation model, commonly used by the VANET community, in order to present a more realistic vehicular environment [14]. Parameter $\mathrm{m}$ for this propagation model is set to 0.7. Moreover, each point in the figures that follow represents the average of 10 independent simulation experiments in which the simulation time is 300 seconds. Table 1 summarizes the simulation parameters.

The urban scenario represents an area of $1,500 \times 1,500 \mathrm{~m}^{2}$ that is obtained by using digital maps freely available in OpenStreetMap [15] from the downtown area of Valencia (Spain) with real obstacles.

\subsection{Result and Analysis}

Figure 1 shows a clear packet delivery ratio improvement for DBM-ACW in comparison to the IEEE 802.11. This improvement was achieved by adapting the CW size based on the channel history. As a consequence, it reduces CW size variations since increasing the $\mathrm{CW}$ size starting from $C W_{\min }$ is no longer required to find the optimal $\mathrm{CW}$ size. This mechanism allows decreasing the number of retransmissions and, consequently, the number of dropped packets. Furthermore, DBM-ACW outperforms HBCWC by not resetting the CW size to the minimum CW size when the CS array contains two consecutive zeros before a successful transmission (Algorithm 2-Operation 9). The average number of MAC collisions, shown in Figure 2, offers a hint on how to achieve improvements in terms of PDR. As can be observed, the optimal CW size for DBM-ACW was chosen so that it decreases the probability of picking the same backoff value, and, consequently, the number of collisions is also reduced.

Our approach achieves a lower end-to-end delay compared to the IEEE 802.11, as depicted in Figure 3. Although IEEE 802.11 cannot guarantee a delay boundary, DBM-ACW does not show a delay increase when the number of 


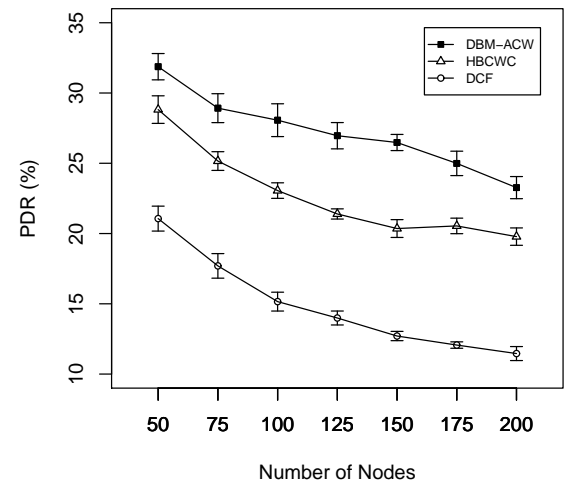

Fig. 1: PDR for the urban scenario.

nodes is larger than 175 , which can help to mitigate the unbounded delay problem in the IEEE 802.11 standard. DBM-ACW gradually decreases the CW, and it does not reset the $\mathrm{CW}$ to $C W_{\min }$ (Algorithm 2-Operation 9), as occurs with IEEE 802.11, thereby increasing the MAC layer delay. However, as a result of decreasing the number of collisions, this increase in terms of MAC layer delay does not have a negative impact in terms of end-to-end delay. Figure 3 evidences the differences between our approach and HBCWC, which are further clarified in Table 2. DBM-ACW is able to achieve improvements in terms of end-to-end delay, as well as improved standard deviation values for delay when comparing DBM-ACW to HBCWC, as shown in Table 2.

Table 2: Standard Deviation of delays for the urban scenario.

\begin{tabular}{llllllll}
\hline & $\mathbf{5 0}$ & $\mathbf{7 5}$ & $\mathbf{1 0 0}$ & $\mathbf{1 2 5}$ & $\mathbf{1 5 0}$ & $\mathbf{1 7 5}$ & $\mathbf{2 0 0}$ \\
\hline \hline DBM-ACW & 0.42 & 0.45 & 0.46 & 0.47 & 0.49 & 0.52 & 0.53 \\
HBCWC & 0.44 & 0.44 & 0.48 & 0.50 & 0.55 & 0.54 & 0.53 \\
\hline
\end{tabular}

Overall, our approach improves the PDR by $47 \%$, and the end-to-end delay by $16 \%$ when compared with the IEEE 802.11 DCF, and the PDR improves by $16 \%$ in comparison with HBCWC.

\section{Conclusion}

This paper presents DBM-ACW, a new IEEE 802.11-based MAC protocol, which controls the $\mathrm{CW}$ size based on a network density estimation. In each vehicle, 


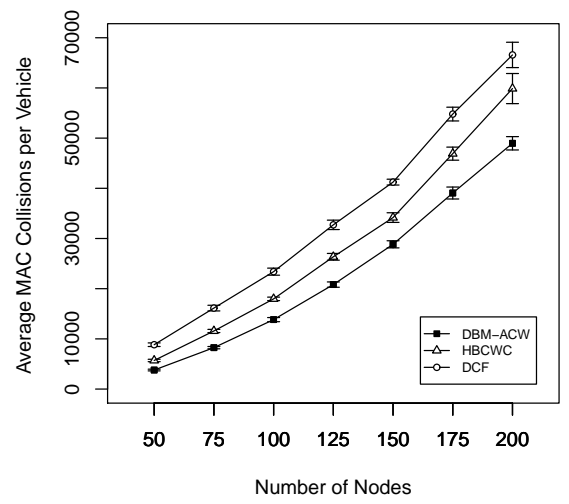

Fig. 2: Average number of collisions for the urban scenario.

transmission trials are stored as an array which is used to determine the optimal contention window value. Extensive simulations using OMNeT++ in urban scenarios prove that our scheme has better overall performance compared with the IEEE 802.11 DCF and HBCWC in terms of PDR, end-to-end delay and average number of collisions in both scenarios.

As future work, we will study the effect of deploying RSUs on the performance of our scheme. Also, we will dynamically adapt the algorithm's parameters for each specific network scenario.

\section{Acknowledgment}

This work was partially supported by the Ministerio de Ciencia e Innovación, Spain, under Grant TIN2011-27543-C03-01.

\section{References}

1. Booysen, M.J.; Zeadally, S.; van Rooyen, G.-J., "Survey of media access control protocols for vehicular ad hoc networks," Communications, IET , vol.5, no.11, pp.1619,1631, July 222011.

2. Kenney, J.; "Standards and regulations," in Hartenstein, H., Laberteaux, K.P. (Eds.): "VANET: vehicular applications and inter-networking technologies" (Wiley, 2010), Ch. 10, pp. 365428.

3. Stanica, R.; Chaput, E.; Beylot, A.-L., "Enhancements of IEEE 802.11p Protocol for Access Control on a VANET Control Channel," Communications (ICC), 2011 IEEE International Conference on , vol., no., pp.1,5, 5-9 June 2011.

4. Calafate, C.T.; Fortino, G.; Fritsch, S.; Monteiro, J.; Cano, J.; Manzoni, P., "An efficient and robust content delivery solution for IEEE 802.11p vehicular environments," Journal of Network and Computer Applications, vol.35, no.2, pp.753,762, March. 2012. 


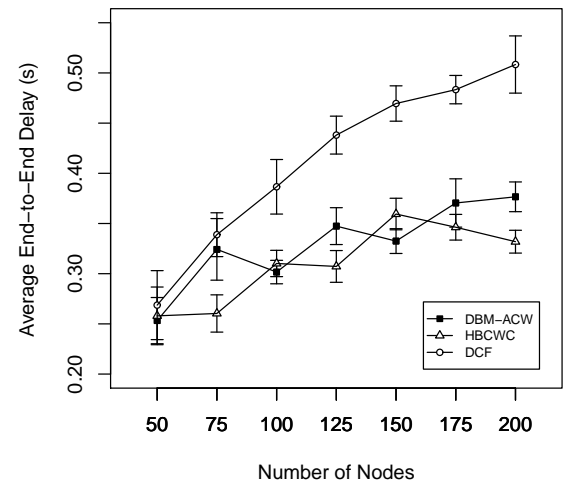

Fig. 3: Average end-to-end delay for the urban scenario.

5. Cali, F.; Conti, M.; Gregori, E., "Dynamic tuning of the IEEE 802.11 protocol to achieve a theoretical throughput limit," Networking, IEEE/ACM Transactions on , vol.8, no.6, pp.785,799, Dec 2000.

6. Wu, H.; Cheng, S.; Peng, Y.; Long, K.; Ma, J., "IEEE 802.11 distributed coordination function (DCF): analysis and enhancement," Communications, 2002. ICC 2002. IEEE International Conference on , vol.1, no., pp.605,609, 2002.

7. Balador, A.; Movaghar, A.; Jabbehdari, S., "History based contention window control in ieee 802.11 mac protocol in error prone channel," Journal of Computer Science, vol.6, no.2, pp.205,209, 2010.

8. Chrysostomou, C.; Djouvas, C.; Lambrinos, L., "Applying adaptive QoS-aware medium access control in priority-based vehicular ad hoc networks," Computers and Communications (ISCC), 2011 IEEE Symposium on , vol., no., pp.741,747, June 28 2011-July 12011.

9. Jang, H.-C.; Feng, W.-C., "Network Status Detection-Based Dynamic Adaptation of Contention Window in IEEE 802.11p," Vehicular Technology Conference (VTC 2010-Spring), 2010 IEEE 71st, vol., no., pp.1,5, 16-19 May 2010.

10. Website: http://www.omnetpp.org/

11. Website: http://inet.omnetpp.org/

12. Behrisch, M.; Bieker, L.; Erdmann, J.; Krajzewicz, D., SUMO - Simulation of Urban MObility: An Overview In: SIMUL 2011, The Third International Conference on Advances in System Simulation, 2011.

13. Baguena, M.; Tornell, S.; Torres, A.; Calafate, C.T.; Cano, J.C.; Manzoni, P., "VACaMobil: VANET Car Mobility Manager for OMNeT++," IEEE International Conference on Communications 2013 3rd IEEE International Workshop on Smart Communication Protocols and Algorithms (SCPA 2013), June 2013, Budapest, Hungary.

14. Baguena, M.; Calafate, C.T.; Cano, J.; Manzoni, P., "Towards realistic vehicular network simulation models," Wireless Days (WD), 2012 IFIP , vol., no., pp.1,3, 21-23 Nov. 2012.

15. Website: http://www.openstreetmap.org/ 